\title{
ON THE INFINITE DIVISIBILITY OF THE VON MISES DISTRIBUTION
}

\author{
TOBY LEWIS
}

(Received 24 March 1975; revised 14 July 1975)

\begin{abstract}
It is shown, by use of a Bochner-type condition for infinite divisibility, that the von Mises distribution is infinitely divisible for some values of the concentration parameter $k$, certainly for $k<0.16$.
\end{abstract}

\section{Introduction}

An angular random variable $X$, centred without loss of generality about 0 . has the von Mises distribution if its probability density function (p.d.f.) is of the form

$$
\begin{gathered}
f(x)=\left\{1 / 2 \pi I_{0}(k)\right\} \exp (k \cos x), \\
x \in(-\pi, \pi], \quad k>0 .
\end{gathered}
$$

This distribution depends on a single parameter $k$, the concentration parameter Its characteristic function (c.f.), defined (as with any distribution on the circle' only for integer values of the argument $t$, is

$$
\phi(t)=E\left(e^{i x x}\right)=I_{t}(k) / I_{0}(k) .
$$

Here $I_{t}(k)$ denotes the Bessel function of order $t$ with imaginary argument. Fol a detailed account of the von Mises distribution and its properties see, fol example, Mardia (1972).

In a recent paper by the present author (Lewis, 1975), it was asserted tha the von Mises distribution was infinitely divisible (i.d.) at any rate for sufficiently small values of the concentration parameter $k$. The proof given was however no valid, since the key inequality on which it rested, given below as (3), was no shown to hold uniformly with respect to $k$. The object of the present paper is tc give a valid proof of the assertion, and to show additionally that the range $o$ values of $k$ for which the infinite divisibility property holds good extend: certainly to $k=0.16$. 


\section{A Bochner-type condition for infinite divisibility}

We first establish, as a particular case of a theorem due to Johansen (1966), I necessary and sufficient condition for the infinite divisibility of an even listribution (whether on the line or the circle). For present purposes, let $\phi(t)$ be I real-valued, and therefore even, c.f. on the circle, and suppose further that it is trictly positive for all integer values of $t$. Write

$$
\lambda(t)=-\ln \phi(t) .
$$

Jearly $\lambda(t)$ is real, even and non-negative $\forall$ integer $t$, and $\lambda(0)=0$. Then a lecessary and sufficient condition for $\phi(t)$ to be i.d. is

$$
\sum_{i=1}^{n} \sum_{j=1}^{n} a_{i} a_{i}\left(\lambda_{i 0}+\lambda_{j 0}-\lambda_{i j}\right) \geqq 0
$$

$\forall$ integer $n ; \forall$ real $a_{1}, \cdots, a_{n} ; \forall$ integer-valued $t=\left(t_{0}, t_{1}, \cdots, t_{n}\right)^{\prime}$, where $\lambda_{i j}$ lenotes $\lambda\left(t_{i}-t_{j}\right)$ for short; ir equivalently, if

$$
m_{i j}=\lambda_{i 0}+\lambda_{j 0}-\lambda_{i j}
$$

he matrix

$$
\boldsymbol{M}=\left\{\boldsymbol{m}_{i j}\right\}
$$

$s$ non-negative definite $\forall n, \forall$ integer-valued $t$. To keep the present paper self ontained we give a proof of this result, but would emphasise that it can be lerived as a particular case of theorem 2 of Johansen (1966), p. 305, combined vith Johansen's inequalities 2 and 3 of lemma 1 (ibid).

$$
\phi \text { will be i.d. if and only if }\{\phi(t)\}^{r} \text { is a c.f. } \forall r>0 \text {, }
$$

Ind this condition is equivalent to the condition that

$\{\phi(t)\}^{\prime}$ is a c.f. $\forall r \in\left(0, r^{*}\right]$ for any positive $r^{*}$ however small, ince a distribution with c.f. $\{\phi(t)\}^{s}$ for any $s>r^{*}$ can be generated as the :onvolution of distributions whose c.f.s. have the form $\{\phi(t)\}^{r}$ with $r \in\left(0, r^{*}\right]$. Nrite $h(t)=\{\phi(t)\}$. By Bochner's theorem, $h$ is a c.f. if and only if, for any sositive integer $n$, for any vector $c=\left(c_{0}, c_{1}, \cdots, c_{n}\right)^{\prime}$ of complex quantities, and or any vector $t=\left(t_{0}, t_{1}, \cdots, t_{n}\right)^{\prime}$ of integers,

$$
\sum_{i=0}^{n} \sum_{j=0}^{n} c_{i} \bar{c}_{j} h\left(t_{i}-t_{j}\right) \geqq 0 \text {. }
$$

ince $h$ is a real-valued and even, this condition reduces, on putting $c_{j}=a_{j}+i b_{j}$, $:=a+i b$, to 


$$
\begin{gathered}
\sum_{i=0}^{n} \sum_{j=0}^{n}\left(a_{i} a_{j}+b_{i} b_{j}\right) h\left(t_{i}-t_{j}\right) \geqq 0 \\
\forall n, \forall \text { real } a ; b, \forall t,
\end{gathered}
$$

or equivalently to

$$
\sum_{i=0}^{n} \sum_{j=0}^{n} a_{i} a_{j} h\left(t_{i}-t_{j}\right) \geqq 0
$$

$\forall n, \forall$ real a, $\forall t$

That is, $\phi$ is i.d. if and only if, given any positive integer $n$, any vector $a$ of real quantities $a_{0}, a_{1}, \cdots, a_{n}$, any vector $t$ of integers $t_{0}, t_{1}, \cdots, t_{n}$, there exists a positive $r^{*}$ (which may depend on $n, a, t$ ) such that

$$
\sum_{i=0}^{n} \sum_{i=0}^{n} a_{i} a_{j}\left\{\phi\left(t_{i}-t_{i}\right)\right\}^{r} \geqq 0 \quad \forall r \in\left(0, r^{*}\right]
$$

Suppose that $\phi$ is i.d. Then, from (6),

$$
\sum_{i=0}^{n} \sum_{j=0}^{n} a_{i} a_{i} \exp \left(-r \lambda_{i j}\right) \geqq 0
$$

or

$$
\left(\sum_{i=0}^{n} a_{i}\right)^{2}-\sum_{i=0}^{n} \sum_{j=0}^{n} a_{i} a_{i}\left(1-e^{-r \lambda_{i j}}\right) \geqq 0 \quad \forall n, a, t, \forall r \in\left(0, r^{*}\right]
$$

In particular, taking $a_{0}=-\left(a_{1}+\cdots+a_{n}\right)$,

$$
\begin{gathered}
g(r) \equiv \sum_{i=1}^{n} \sum_{j=1}^{n} a_{i} a_{j}\left\{\left(1-e^{-r \lambda_{0 i}}\right)+\left(1-e^{-r \lambda_{0 i}}\right)-\left(1-e^{-r \lambda_{i j}}\right)\right\} \geqq 0 \\
\forall a_{1}, \cdots, a_{n} ; t_{0}, t_{1}, \cdots, t_{n} ; \forall r \in\left(0, r^{*}\right] .
\end{gathered}
$$

Now $g(r)$ is a continuous and differentiable function of $r$, and $g(0)=0$, hence $g^{\prime}(0) \geqq 0$, i.e. (3) is a necessary condition for $\phi$ to be i.d.

Suppose that (3) holds. Then $\phi$ is i.d. For, given any positive integer $n$, any vector $a$ of real quantities $a_{0}, a_{1}, \cdots, a_{n}$, and any vector $t$ of integers $t_{0}, t_{1}, \cdots, t_{n}$, the expression on the l.h.s. of (6)

$$
\sum_{i=0}^{n} \sum_{j=0}^{n} a_{i} a_{j}\left\{\phi\left(t_{i}-t_{j}\right)\right\}^{r}
$$

is equal to 


$$
\begin{aligned}
& \sum_{i=0}^{n} \sum_{j=0}^{n} a_{i} a_{i} e^{-r \lambda_{i j}} \\
& \geqq \sum_{i=0}^{n} \sum_{j=0}^{n} a_{i} a_{i}\left(1-r \lambda_{i j}\right), \text { since } e^{-x}>1-x \text { for } x>0, \\
& \quad=\left(\sum_{i=0}^{n} a_{i}\right)^{2}-r \sum_{i=0}^{n} \sum_{j=0}^{n} a_{i} a_{j} \lambda_{i j}=\psi(r), \text { say } .
\end{aligned}
$$

If $\sum_{i=0}^{n} a_{i} \neq 0, \sum_{i=0}^{n} \sum_{i=0}^{n} a_{i} a_{j} \lambda_{i j}=0, \quad \psi(r)>0 \quad \forall r$;

if $\sum_{i=0}^{n} a_{i} \neq 0, \sum_{i=0}^{n} \sum_{j=0}^{n} a_{i} a_{i} \lambda_{i j} \neq 0, \quad \psi(r) \geqq 0$ for $r \in\left(0, r^{*}\right]$

where

$$
r^{*}=\left(\sum_{i=0}^{n} a_{i}\right)^{2} /\left|\sum_{i=0}^{n} \sum_{j=0}^{n} a_{i} a_{i} \lambda_{i j}\right|
$$

and finally if $\sum_{i=0}^{n} a_{i}=0, \psi(r)$ is equal to

$$
r \sum_{i=1}^{n} \sum_{j=1}^{n} a_{i} a_{j}\left(\lambda_{i 0}+\lambda_{j 0}-\lambda_{i j}\right)
$$

which, for any $r>0$, is non-negative from (3).

Thus in all cases condition (6) is satisfied, i.e. (3) is a sufficient condition for $\phi$ to be i.d.

\section{Main result}

We now particularise to the von Mises distribution (1).

THEOREM. The von Mises distribution is infinitely divisible for some values of the concentration parameter $k$, certainly for $k<0.16$.

Proof. The c.f. of the distribution is

$$
\phi(t)=I_{i}(k) / I_{0}(k) \quad(t=0, \pm 1, \pm 2, \cdots),
$$

which is even (w.r.t.t) and strictly positive $\forall k>0, \forall$ integer $t$. For non-negative integer $t$ we have

$$
I_{t}(k)=\frac{\left(\frac{1}{2} k\right)^{t}}{t !}\left\{1+\frac{\frac{1}{4} k^{2}}{1 !(t+1)}+\frac{\left(\frac{1}{4} k^{2}\right)^{2}}{2 !(t+1)(t+2)}+\cdots\right\},
$$

thus

$$
\begin{aligned}
\ln I_{t}(k) & =-t \xi-\ln t !+f_{t} \quad \text { where } \\
\xi & =\ln (2 / k) \text { and }
\end{aligned}
$$


(9)

$$
f_{t}=\ln \left\{1+\frac{\frac{1}{4} k^{2}}{1 !(t+1)}+\frac{\left(\frac{1}{4} k^{2}\right)^{2}}{2 !(t+1)(t+2)}+\cdots\right\}
$$

Clearly $f_{0}>f_{1}>f_{2}>\cdots$ and

$$
0<f_{t}<\ln \exp \frac{\frac{1}{4} k^{2}}{t+1}=\frac{k^{2}}{4(t+1)} .
$$

We have $\lambda(t)=-\ln \phi(t)=t \xi+\ln t !-f_{t}+f_{0}$. For the application of condition (3) we can without loss of generality take $t_{0}, t_{1}, \cdots, t_{n}$ to be respectively $0,1, \cdots, n$. In the notation of (4),

$$
m_{i j}=2 \xi \min (i, j)+\ln \frac{i ! j !}{|i-j| !}-f_{i}-f_{j}+f_{|i-j|}+f_{0} .
$$

The quadratic form in (3), $Q$ say, can be written as

$$
Q=\left(a_{1}, a_{2}, \cdots ; a_{n}\right) M\left(a_{1}, a_{2}, \cdots, a_{n}\right)^{\prime} ;
$$

suppose that instead of the arbitrary vector $\left(a_{1}, a_{2}, \cdots, a_{n}\right)$ we use equivalently the arbitrary vector $\left(u_{1}, u_{2}, \cdots, u_{n}\right)=u^{\prime}$, given by

$$
\left(u_{1}, u_{2}, \cdots, u_{n}\right)^{\prime}=T\left(a_{1}, a_{2}, \cdots, a_{n}\right)^{\prime}
$$

where $T$ is the non-singular $n \times n$ matrix

$$
\boldsymbol{T}=\left[\begin{array}{ccccc}
1 & 1 & 1 & \ldots & 1 \\
0 & 1 & 1 & \ldots & 1 \\
0 & 0 & 1 & \ldots & 1 \\
. & . & . & \ldots & \cdot \\
0 & 0 & 0 & \ldots & 1
\end{array}\right]
$$

$$
\text { Then } \boldsymbol{T}^{-1}=\left[\begin{array}{rrrrrr}
1 & -1 & 0 & 0 & \ldots & 0 \\
0 & 1 & -1 & 0 & \ldots & 0 \\
0 & 0 & 1 & -1 & \ldots & 0 \\
. & . & . & . & \ldots & . \\
0 & 0 & 0 & 0 & \ldots & 1
\end{array}\right]
$$

and $Q=\boldsymbol{u}^{\prime}\left(\boldsymbol{T}^{-1}\right)^{\prime} \boldsymbol{M} \boldsymbol{T}^{-1} \boldsymbol{u}$,

i.e. ,

$$
Q=\boldsymbol{u}^{\prime} \boldsymbol{H} \boldsymbol{u}
$$

where $\boldsymbol{H}=\left(\boldsymbol{T}^{-1}\right)^{\prime} \boldsymbol{M} \boldsymbol{T}^{-1}$. The typical term $h_{i j}$ of this matrix $\boldsymbol{H}$ is given by

$$
\begin{aligned}
h_{i j}=m_{i j}- & m_{i-1, j}-m_{i, j-1}+m_{i-1, j-1} \\
& (i=1, \cdots, n ; j=1, \cdots, n)
\end{aligned}
$$


where $m_{i 0}$ and $m_{0 i}$ are to be taken as zero, consistently with (4). From (11) and (13),

$$
h_{\mathrm{ii}}=2 \xi \quad+2\left(f_{0}-f_{1}\right) \quad=\theta_{0}, \text { say, }
$$

and for $i \neq j$, writing $|i-j|=d$,

$$
h_{i j}=\ln \left(\frac{d+1}{d}\right)-\left(f_{d-1}-2 f_{d}+f_{d+1}\right)=\theta_{d}, \text { say. }
$$

$\boldsymbol{H}$ is a Toeplitz (or Laurent) matrix (see, for example, Grenander and Szegö (1958)), with all elements on the pairs of diagonals $|i-j|=d$ having the same value $\theta_{d}$. We have to show that $\boldsymbol{H}$ is non-negative definite uniformly w.r.t.n, i.e. that there exists a $k^{*}>0$ independent of $n$ such that the quadratic form $Q=\boldsymbol{a}^{\prime} \boldsymbol{H} \boldsymbol{u}$ satisfies condition (3) (with $u^{\prime} \mathrm{s}$ read for $a$ 's) $\forall k \in\left(0, k^{*}\right]$. In considering the sign of $Q$ as $u$ varies, it is convenient to adjoin any suitable norming equation on the $u$ 's, and we shall choose

$$
\boldsymbol{u}^{\prime} \boldsymbol{u}=\sum_{i=1}^{n} u_{i}^{2}=1
$$

We require the four following lemmas.

LemMA A. Suppose $\boldsymbol{u}^{\prime}=\left(u_{1}, u_{2}, \cdots, u_{n}\right)$ is a vector of real quantities, with $\boldsymbol{u}^{\prime} \boldsymbol{u}=1$. Write

$$
\sum_{i=1}^{n-p} u_{i} u_{i+p}=s_{p} \quad(p=0,1, \cdots, n-1) .
$$

Then for $p=1,2, \cdots,(n-2) / 2$ [ $n$ even] or $(n-1) / 2$ [ $n$ odd ], the point $\left(s_{p}, s_{n-p}\right)$ cannot lie outside the square in the $\left(s_{p}, s_{n-p}\right)$ plane with vertices $(1,0),(0,1)$, $(-1,0),(0,-1)$; and if $h_{1}, \cdots, h_{n-1}$ are any real quantities,

$$
\left|h_{p} s_{p}+h_{n-p} s_{n-p}\right| \leqq \max \left(\left|h_{p}\right|,\left|h_{n-p}\right|\right) .
$$

For even $n$ and $p=n / 2$, the corresponding result is

$$
\left|h_{p} s_{p}\right| \leqq \frac{1}{2}\left|h_{p}\right| \text {. }
$$

Proof. In Schwarz's inequality

$$
\left(\sum_{i=1}^{n} \alpha_{i} \beta_{i}\right)^{2} \leqq \sum_{i=1}^{n} \alpha_{i}^{2} \sum_{i=1}^{n} \beta_{i}^{2}
$$

take

$$
\alpha_{1}, \cdots, \alpha_{n}=u_{1}, \cdots, u_{n-p} ; \quad u_{n-p+1}, \cdots, u_{n}
$$

and

$$
\beta_{1}, \cdots, \beta_{n}=u_{1+p}, \cdots, u_{n} ; \quad u_{1}, \cdots, u_{p}
$$


then

$$
\left(s_{p}+s_{n-p}\right)^{2} \leqq\left(\sum_{i=1}^{n} u_{i}^{2}\right)^{2}=1 ;
$$

similarly, take the same $\alpha$ 's and

$$
\beta_{1}, \cdots, \beta_{n}=u_{1+p}, \cdots, u_{n} ;-u_{1}, \cdots,-u_{p}
$$

then

$$
\left(s_{p}-s_{n-p}\right)^{2} \leqq 1 .
$$

The extreme values of $h_{p} s_{p}+h_{n-p} s_{n-p}$ are attained when a straight line of the parallel system $h_{p} s_{p}+h_{n-p} s_{n-p}=$ constant passes through a vertex of the square with vertices $( \pm 1,0),(0, \pm 1)$.

Lemma B. Suppose that $\boldsymbol{H}=\left\{h_{i j}\right\}$ is a real $n \times n$ Toeplitz matrix with elements $h_{i j}=h_{d}$ for $|i-j|=d$. Suppose that a non-increasing sequence of positive constants $g_{1}, \cdots, g_{n-1}$ exists, $g_{1} \geqq g_{2} \geqq \cdots \geqq g_{n-1}>0$, such that

$$
\left|h_{p}\right| \leqq g_{p}, p=1,2, \cdots, n-1 .
$$

Then if $Q$ is the quadratic form $\boldsymbol{u}^{\prime} \boldsymbol{H} \boldsymbol{u}$, where $\boldsymbol{u}^{\prime} \boldsymbol{u}=1$,

$$
\left|Q-h_{0}\right| \leqq 2\left\{g_{1}+g_{2}+\cdots+g_{(n-1) / 2}\right\} \quad \text { (nodd) }
$$

or

$$
\leqq 2\left\{g_{1}+g_{2}+\cdots+g_{(n-2) / 2}\right\}+g_{n / 2} \quad \text { (n even) } .
$$

Proof. Since $Q=h_{0}+2\left(h_{1} s_{1}+h_{2} s_{2}+\cdots+h_{n-1} s_{n-1}\right)$, the result follows from Lemma $A$.

Lemma C. The $n \times n$ Toeplitz matrix $\boldsymbol{H}$ with $h_{0}=1, \quad h_{d}=1 / C d$ $(d=1,2, \cdots, n-1)$, is positive definite for $C$ sufficiently large, certainly for $C \geqq 2(1+\ln 2)=3.386$.

Proof. Given $n-1$ quantities $\rho_{1}, \rho_{2}, \cdots, \rho_{n-1}$, write

$$
\begin{aligned}
A(z) & =1+\sum_{d=1}^{n-1} \rho_{d}\left(z^{d}+z^{-d}\right) \\
& =B(y), \text { where } y=z+z^{-1} .
\end{aligned}
$$

$B(y)$ is a polynomial in $y$ of degree $n-1$. A theorem of Wold's (Wold (1954), p. 154; Kendall and Stuart (1968), p. 415) states that $\rho_{1}, \cdots, \rho_{n-1}$ can be the autocorrelations of a moving average of extent $n$ of some random series, if and only if $B(y)$ has no real zero of odd multiplicity in the interval $-2<y<2$. We shall show that, when $\rho_{d}=1 / C d$ and $C \geqq 2(1+\ln 2), B(y)$ satisfies this condition. We have 


$$
\begin{aligned}
& A(z)=1+\frac{1}{C} \sum_{d=1}^{n-1} \frac{z^{d}+z^{-d}}{d} \\
& A^{\prime}(z)=\frac{1}{C} \sum_{d=1}^{n-1}\left(z^{d-1}-z^{-d-1}\right)=\frac{\left(z^{n-1}-1\right)\left(z^{n}-1\right)}{C(z-1) z^{n}} \\
& B^{\prime}(y)=\frac{\left(z^{n-1}-1\right)\left(z^{n}-1\right)}{C z^{n-2}(z-1)^{2}(z+1)} .
\end{aligned}
$$

We now show that $B(y)>0$ for $y \in(-2,2)$. Write $z=e^{2 i \theta}, y=2 \cos 2 \theta$; the $\theta$-interval $(0, \pi / 2)$ maps into the $y$-interval $(-2,2)$. If $B(y)=F(\theta)$, we have

$$
\begin{aligned}
F\left(\frac{\pi}{2}\right) & =B(-2)=A(-1)=1-\frac{2}{C}\left(1-\frac{1}{2}+\frac{1}{3}-\cdots+\frac{(-)^{n}}{n-1}\right) \\
& \geqq 1-\frac{2}{C} \text { for } n>1 .
\end{aligned}
$$

Also from (17)

$$
\frac{d F(\theta)}{d \theta}=-\frac{4}{C} \frac{\sin (n-1) \theta \sin n \theta}{\sin \theta}=-\frac{2}{C} \frac{\cos \theta-\cos (2 n-1) \theta}{\sin \theta},
$$

hence

$$
\frac{C}{2}\left\{F(\theta)-F\left(\frac{\pi}{2}\right)\right\}=\int_{\theta}^{\pi / 2} \frac{\cos u}{\sin u} d u-\int_{\theta}^{\pi / 2} \frac{\cos (2 n-1) u}{\sin u} d u
$$

For $0<\theta \leqq u \leqq \pi / 2, \sin u$ is positive; hence

$$
\begin{aligned}
\frac{C}{2}\{F(\theta)- & \left.F\left(\frac{\pi}{2}\right)\right\} \geqq \int_{\theta}^{\pi / 2} \frac{\cos u}{\sin u} d u-\int_{\theta}^{\pi / 2} \frac{1}{\sin u} d u \\
& =-\int_{\theta}^{\pi / 2} \tan \frac{u}{2} d u=2\left[\ln \cos \frac{u}{2}\right]_{\theta}^{\pi / 2}=-\ln 2-2 \ln \cos \frac{\theta}{2} \geqq-\ln 2 .
\end{aligned}
$$

Therefore $F(\theta)>1-2 / C-(2 / C) \ln 2$. It follows that, if $C \geqq 2(1+\ln 2)$, $B(y)>0 \forall y \in(-2,2)$. Wold's theorem therefore applies, and $\boldsymbol{H}$ is a correlation matrix, and accordingly positive definite, for all $n \geqq 2$.

LeMma D. For the quantities $f_{0}, f_{1}, f_{2}, \cdots$ defined by (9),

$$
\left|f_{d-1}-2 f_{d}+f_{d+1}\right|<\frac{u}{d(d+1)} \quad(d=1,2, \cdots)
$$

where

$$
u=\frac{1}{4} k^{2}
$$


Proof. Write

$$
E_{d}=\exp \left(f_{d}\right)=\sum_{r=0}^{\infty} \frac{u^{r}}{r !} \frac{d !}{(d+r) !} \quad(d=0,1, \cdots) ;
$$

$\left\{f_{d}\right\}, \quad\left\{E_{d}\right\}$ are decreasing sequences of positive quantities. Since $I_{d-1}(k)-I_{d+1}(k)=(2 d / k) I_{d}(k)$, it follows that

$$
E_{d-1}-E_{d}=\frac{u}{d(d+1)} E_{d+1}
$$

For any $\alpha, \beta$ satisfying $\alpha>\beta>0$,

$$
0<e^{\alpha}-e^{\beta}=(\alpha-\beta)\left(1+\frac{\alpha+\beta}{2 !}+\frac{\alpha^{2}+\alpha \beta+\beta^{2}}{3 !}+\cdots\right) .
$$

This

$$
>(\alpha-\beta)\left(1+\frac{2 \beta}{2 !}+\frac{3 \beta^{2}}{3 !}+\cdots\right)=(\alpha-\beta) e^{\beta}
$$

and similarly $<(\alpha-\beta) e^{\alpha}$. Hence

$$
\frac{e^{\alpha}-e^{\beta}}{e^{\alpha}}<\alpha-\beta<\frac{e^{\alpha}-e^{\beta}}{e^{\beta}} .
$$

From (19), for $d \geqq 1$,

$$
\begin{aligned}
\left(f_{d-1}-f_{d}\right)-\left(f_{d}-f_{d+1}\right) & >\frac{E_{d-1}-E_{d}}{E_{d-1}}-\frac{E_{d}-E_{d+1}}{E_{d+1}} \\
& >-\frac{E_{d}-E_{d+1}}{E_{d+1}}=-\frac{u}{(d+1)(d+2)} \frac{E_{d+2}}{E_{d+1}}, \text { from (18), } \\
& >-\frac{u}{(d+1)(d+2)}>-\frac{u}{d(d+1)}
\end{aligned}
$$

Again

$$
\begin{aligned}
\left(f_{d-1}-f_{d}\right)-\left(f_{d}-f_{d+1}\right) & <\frac{E_{d-1}-E_{d}}{E_{d}}-\frac{E_{d}-E_{d+1}}{E_{d}} \\
& <\frac{E_{d-1}-E_{d}}{E_{d}}=\frac{u}{d(d+1)} \frac{E_{d+1}}{E_{d}}<\frac{u}{d(d+1)} .
\end{aligned}
$$

This proves the required result.

Reverting to equation (15), we have, for $x>0$,

$$
x-\frac{x^{2}}{2}<\ln (1+x)<x
$$


lence for $d=1,2, \cdots$

$$
\frac{1}{d}-\frac{1}{2 d^{2}}<\ln \frac{d+1}{d}<\frac{1}{d}
$$

$\mathrm{t}$ follows from Lemma $D$ that

20)

$$
\left|\theta_{d}-\frac{1}{d}\right|<\frac{1}{2 d^{2}}+\frac{u}{d(d+1)}
$$

From (12), (14), (16) and (20) we can now write

$$
\begin{aligned}
Q=\theta_{0}-C+u & {\left[\begin{array}{lllll}
C & 1 & \frac{1}{2} & \frac{1}{3} & \ldots \\
1 & C & 1 & \frac{1}{2} & \ldots \\
\frac{1}{2} & 1 & C & 1 & \ldots \\
\cdot & . & . & . & \ldots
\end{array}\right] u } \\
& +u^{\prime}\left[\begin{array}{lllll}
h_{0} & h_{1} & h_{2} & h_{3} & \ldots \\
h_{1} & h_{0} & h_{1} & h_{2} & \ldots \\
h_{2} & h_{1} & h_{0} & h_{1} & \ldots \\
. & . & . & . & \ldots
\end{array}\right] u
\end{aligned}
$$

where $h_{0}=0$ and

$$
\left|h_{p}\right| \leqq \frac{1}{2 p^{2}}+\frac{u}{p(p+1)} \quad(p=1,2, \cdots, n-1) .
$$

The constant $C$ is at choice; assign it the value $2(1+\ln 2)$, then by Lemma $\tau$ the third term on the right of the equation is positive. By Lemma $B$, the nodulus of the fourth term

$$
\begin{aligned}
& \leqq 2\left\{\frac{1}{2.1^{2}}+\frac{1}{2.2^{2}}+\cdots+\frac{u}{1.2}+\frac{u}{2.3}+\ldots\right\} \\
& <\frac{\pi^{2}}{6}+2 u \text { whatever the value of } n .
\end{aligned}
$$

Hence

$$
\begin{aligned}
Q & >2 \xi+2\left(f_{0}-f_{1}\right)-2(1+\ln 2)-\frac{\pi^{2}}{6}-\frac{1}{2} k^{2} \\
& =2\left\{\ln \frac{I_{0}(k)}{I_{1}(k)}-\left(1+\ln 2+\frac{\pi^{2}}{12}+\frac{1}{4} k^{2}\right)\right\} \\
& =2\left\{\ln \frac{I_{0}(k)}{I_{1}(k)}-\left(2.51562+\frac{k^{2}}{4}\right)\right\}
\end{aligned}
$$


This last expression has a unique positive zero $k^{*}=0.161$, and is positive for $k \in\left(0, k^{*}\right)$. $Q$ is thus uniformly positive w.r.t. $n$, and the von Mises distribution infinitely divisible, for all values of $k$ in this range.

\section{Acknowledgements}

I am most grateful to the referee for drawing my attention to Johansen's work.

\section{References}

U. Grenander and G. Szegö (1958), Toeplitz Forms and their Applications (University of California Press, Berkeley and Los Angeles, 1958).

S. Johansen (1966), 'An application of extreme point methods to the representation of infinitely divisible distributions', $Z$. Wahrscheinlichkeitstheorie verw. Geb. 5, 304-316.

M. G. Kendall and A. Stuart (1968), The Advanced Theory of Statistics, Vol. 3 (Griffin, London, 1968).

T. Lewis (1975), 'Probability functions which are proportional to characteristic functions and the infinite divisibility of the von Mises distribution', contribution to Perspectives in Probability and Statistics (Papers in Honour of M. S. Bartlett on the Occasion of his Sixty-Fifth Birthday) ed. J. Gani (Academic Press, London), 19-28.

K. V. Mardia (1972), Statistics of Directional Data (Academic Press, London and New York, 1972).

H. Wold (1954), A Study in the Analysis of Stationary Time Series (Almqvist and Wiksell, Stockholm, 2nd edition, 1954).

Department of Mathematical Statistics,

The University,

Hull,

England. 